\title{
A Novel Geometrical Approach to Learn the Actions of Extraocular Muscles
}

\author{
Jyothilakshmi.T.K ${ }^{1}$, Gautham.B.V. ${ }^{2}$, Sarala.H.S ${ }^{3}$ \\ Associate professor ${ }^{1}$, Department of Anatomy, Postgraduate ${ }^{2,}$ Department of Pharmacology, postgraduate ${ }^{3}$, \\ Department of Anatomy, Kempegowda institute of medical sciences, Bangalore.
}

\begin{abstract}
The actions of the individual extra ocular muscles are best explained to medical students in a lecture format by showing the relationship of each muscle to the axes of the globe and the walls of the bony orbit. The lateral and medial rectus muscles cross only the vertical axis, and consequently, cause only abduction and adduction, respectively. In contrast, the superior and inferior oblique and rectus muscles cross all three axes (vertical, horizontal, and anteroposterior) of the globe. Therefore, concentric contraction of each of these muscles results in three movements; abduction or adduction, elevation or depression, and intorsion or extorsion. The purpose of this article is to present a method of computing the action of each of the extra ocular muscles with reference to the three cardinal axes, for the primary as well as for the secondary positions of the eyeball.
\end{abstract}

Keywords: Extra ocular muscles, eye movements, dimensional action

\section{Introduction}

The eyeball a peripheral organ of vision situated in a skeletal cavity, the orbit not only protects it from injury but also has a more fundamental role in the visual process itself in providing a rigid support and direction to the eyeball and also contributing to the sites of attachment to its external muscles.A setting that permits an accurate positioning of the visual axis by neuromuscular control and it is this that also determines the spatial relationship between the two eyes. All set for binocular vision and conjugate eye movement ${ }^{1}$.

It is the interplay of the recti and the obliquii muscles which move the eyeball. These are the skeletal muscles that take a bony origin from the orbit and get inserted to the eyeball. Medical students often have difficulty in understanding the actions of the extra ocular muscles. It is not always obvious what movements of the pupil are elicited by concentric contraction of an individual muscle.

\section{Listing's Law}

Mathematician, Johannes Benedict Listing, first described three dimensional eye movements in the middle of the $19^{\text {th }}$ century, but never published his hypotheses. Later, the physiologist Hermann von Helmholtz verified the work of listing and coined the name. It is a generally accepted fact, in considering the subject of ocular rotations that the globe rotates in conformity with Listing's law. Listing's law describes the threedimensional orientation of the eye and its axes of rotation. Listing's law has been shown to hold when the head is stationary and upright and gaze is directed toward far targets, i.e., when the eyes are fixating, making saccades, or pursuing moving visual targets. Listing's law describes the movements from any position of gaze and postulates that the globe revolves about an axis perpendicular to a plane cutting through ${ }^{2}$.

The resolution of the action of the individual muscles has been calculated graphically by Maddox, Duke elder and others. However this was done only in 2 dimensions. Marquez devised a scheme for depicting the three dimensional action of the ocular muscles in the primary position.Verrijp computed the relative movements of force about the three cardinal axes and expressed these in a numerical ratio, also for the primary position only, as did the others. Vander hoeve's mathematics is based on the coupling action of muscles rotating about the same axis. Many others have written extensively on some of the geometric considerations of eye movements. ${ }^{1}$ I now propose a simpler selling concept which is easy to learn without any need for relying on too many geometrical details. Here is a novel approach wherein the traditional understanding as given in the standard textbooks is now redefined in terms of the three dimensional axes as in figure 1.

$\mathrm{X}$ is the traditional horizontal axis

$\mathrm{Y}$ is the traditional anteroposterior axis

And $\mathrm{Z}$ is the traditional vertical axis. 


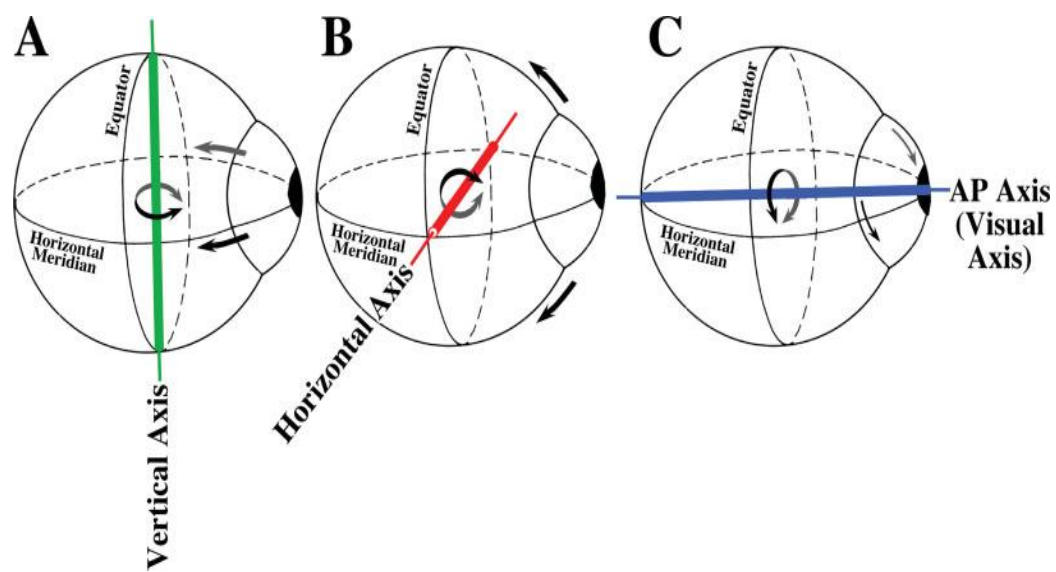

Figure 1- showing the axes of the globe

\section{Axes of the Globe and Actions of Extra Ocular Muscles (Figure 2)}

Here is the scheme for depicting the three dimensional action of each of the ocular muscles. Keeping these axes in the background and by using the methods of solid analytical geometry, a three dimensional system of Cartesian coordinates can be erected in the orbit, the origin of the coordinates being at the centre of rotation of the globe. By convention, the x-axis corresponds to the transverse diameter of the eye, the $\mathrm{y}$-axis to the anteroposterior diameter and the z-axis to the vertical diameter. It is now possible to elaborate on functionalities of the recti and the obliquii muscles. For this purpose the right superior rectus and the superior oblique will be discussed.

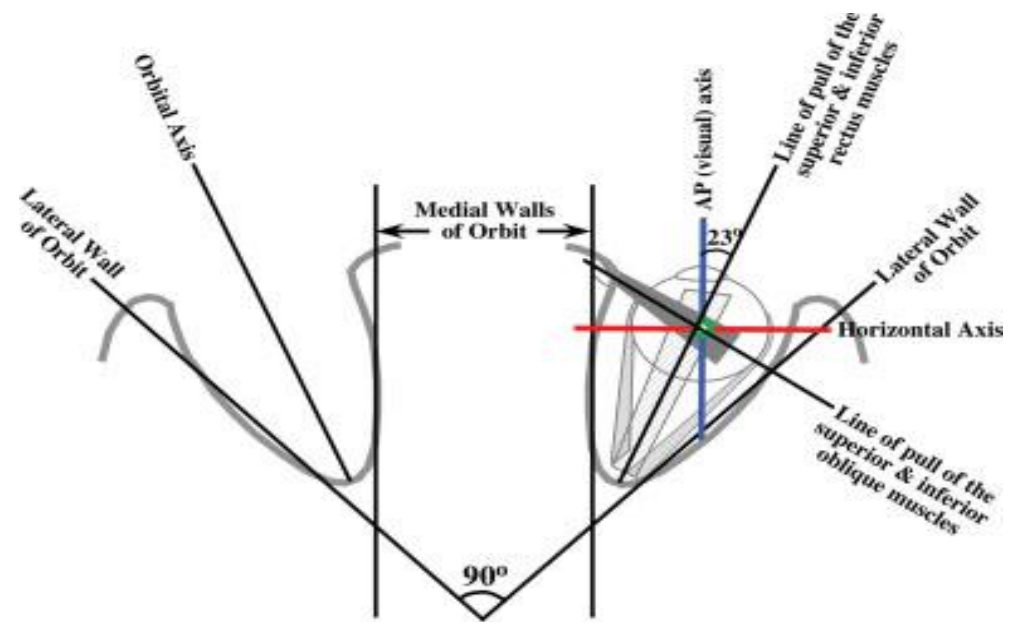

Figure 2 - The position of the globe and extra ocular muscles within the bony orbit is shown

The superior rectus has its own coordinate system and the y' axis corresponds to the long axis of the superior rectus muscle. The eyeball in the primary position and the symmetric coordinate system with respect to the eyeball (always in motion) call this coordinate system as $x^{\prime}$. The angle made by $\mathrm{x}$ and $\mathrm{x}^{\prime}$ is alpha, $\mathrm{y}$ and $\mathrm{y}^{\prime}$ is beta, $\mathrm{z}$ and $\mathrm{z}^{\prime}$ is gamma respectively. In the primary position of the eyeball, it is the existence of these angles that enables the superior rectus to bring about its elevation, intorsion and adduction respectively. In an adducted position of the eyeball the angle beta becomes zero, that is the y axis coincides with the y' (align over one another nullifying the angle between them). Hence the possible action of superior rectus at the $y^{\prime}$ is no more possible and hence intorsion does not occur when the eye is adducted. The rotation of the eyeball is now limited to one plane alone (elevation or depression). Hence superior rectus in an already adducted eye can bring about only elevation.

When it comes to the obliquii muscles the proximal portion of the muscles will be omitted since it is not related to the muscle's direction of pull (fig 3). The long axis of the superior oblique muscle will be from the functional origin where the intermediate tendon of the muscle passes through the trochlea anterior to the globe. The same is true for inferior oblique muscle that is attached to the inferomedial orbital rim and the posterior 
hemisphere of the globe. Both the obliquii similar to recti cross all the three axes of the globe. Therefore the same coordinate system of $x^{\prime}, y^{\prime}$ and $z^{\prime}$ can now be erected in the superior oblique and the actions can be computed.

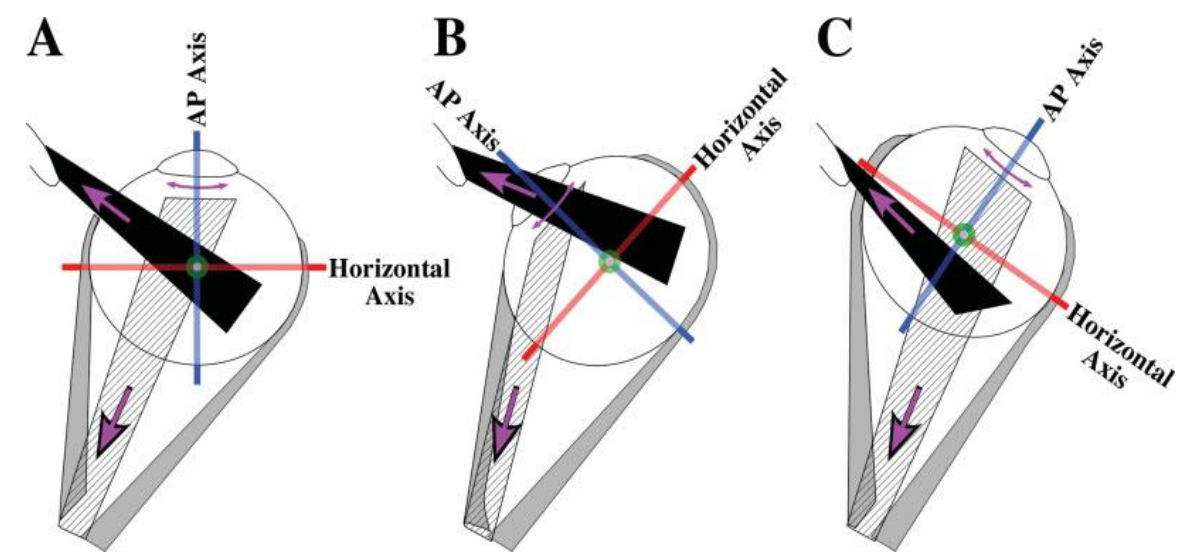

Figure 3 - shows the attachment of superior \& inferior oblique muscles and also the direction of pull.

The actions of the medial and lateral rectus muscles are the easier to understand. These muscles have a proximal attachment to a common tendinous ring in the apex of the orbit and a distal attachment to the globe anterior to the equator. From primary gaze, their line of pull crosses the vertical axis of the globe causing adduction and abduction, respectively (fig 4). These muscles are parallel to the anteroposterior axis of the globe so that they have no effect on intorsion and extorsion. They cross the end of the horizontal axis evenly and are unable to cause elevation and depression.

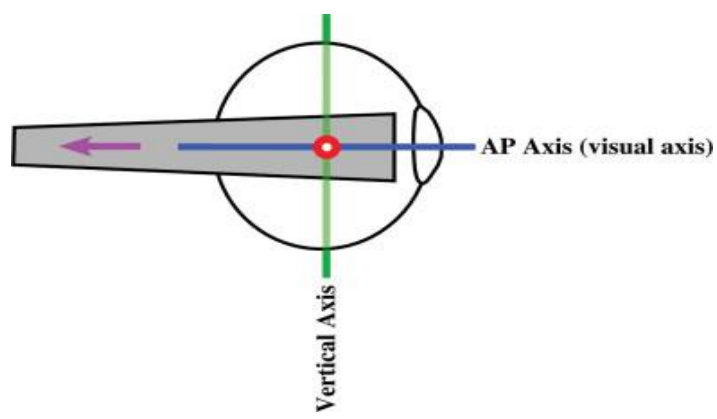

Figure 4 - shows the attachment of Rectii muscles (indicated in grey). Arrow shows direction of pull.

\section{Conclusion}

The axes of the globe are therefore useful in understanding the actions of the extra ocular muscles. Ideally, medical students will not use rote memorization to remember these actions if the simplified approach described above is used in their education. As always, it is the best and novel geometrical approach in learning the actions of extra ocular muscles, and also to grasp the underlying rationale in understanding the functions.

\section{References}

[1]. The action of the extraocular muscles, a method of vector analysis with computations by William E. Krewson, $3^{\text {rd }}$, M.D.

[2]. Listing's law: clinical significance and implications for neurological control, Wong AMF, surv Ophthalmol 2004 (49):563-575

[3]. Current concepts of mechanical and neural factors in ocular motility, Joseph L.Demer,curr opin Neurol 2006(19); 4-13 